\title{
Improvement of Screening Accuracy of Mini-Mental State Examination for Mild Cognitive Impairment and Non-Alzheimer's Disease Dementia by Supplementation of Verbal Fluency Performance
}

\author{
Jee Wook Kim ${ }^{1}$, Dong Young Lee ${ }^{2,3} \bowtie$, Eun Hyun $\mathrm{Seo}^{3}$, Bo Kyung Sohn², Young Min Choe', \\ Shin Gyeom Kim ${ }^{4}$, Shin Young Park ${ }^{5}$, IL Han $\mathrm{Choo}^{6}$, Jong Chul Youn ${ }^{7}$, Jin Hyeong Jhoo ${ }^{8}$, \\ Ki Woong Kim ${ }^{9}$, and Jong Inn $\mathrm{Woo}^{2,3}$ \\ 1Department of Neuropsychiatry, Hallym University Dongtan Sacred Heart Hospital, Hwaseong, Republic of Korea \\ ${ }^{2}$ Department of Neuropsychiatry, Seoul National University Hospital, Seoul, Republic of Korea \\ ${ }^{3}$ Interdisciplinary Program in Cognitive Science, Seoul National University, Seoul, Republic of Korea \\ ${ }^{4}$ Department of Neuropsychiatry, Soonchunhyang University Hospital, Bucheon, Republic of Korea \\ ${ }^{5}$ Department of Neuropsychiatry, Daelim Saint Mary's Hospital, Seoul, Republic of Korea \\ ${ }^{6}$ Department of Neuropsychiatry, Chosun University Hospital, Gwangju, Republic of Korea \\ ${ }^{7}$ Department of Neuropsychiatry, Kyunggi Provincial Hospital for the Elderly, Yongin, Republic of Korea \\ ${ }^{8}$ Department of Neuropsychiatry, Kangwon National University Hospital, Chuncheon, Republic of Korea \\ ${ }^{9}$ Department of Neuropsychiatry, Seoul National University Bundang Hospital, Seongnam, Republic of Korea
}

Objective This study aimed to investigate whether the supplementation of Verbal Fluency: Animal category test (VF) performance can improve the screening ability of Mini-Mental State Examination (MMSE) for mild cognitive impairment (MCI), dementia and their major subtypes.

Methods Six hundred fifty-five cognitively normal (CN), 366 MCI [282 amnestic MCI (aMCI); 84 non-amnestic MCI (naMCI)] and 494 dementia [346 Alzheimer's disease (AD); and 148 non-Alzheimer's disease dementia (NAD)] individuals living in the community were included (all aged 50 years and older) in the study.

Results The VF-supplemented MMSE (MMSE+VF) score had a significantly better screening ability for MCI, dementia and overall cognitive impairment (MCI plus dementia) than the MMSE raw score alone. MMSE+VF showed a significantly better ability than MMSE for both MCI subtypes, i.e., aMCI and naMCI. In the case of dementia subtypes, MMSE+VF was better than the MMSE alone for NAD screening, but not for AD screening.

Conclusion The results support the usefulness of VF-supplementation to improve the screening performance of MMSE for MCI and NAD. Psychiatry Investig 2014;11:44-51

Key Words Mini-Mental State Examination, Verbal fluency, Screening accuracy, Mild cognitive impairment, Non-Alzheimer's disease dementia.

\section{INTRODUCTION}

The Mini-Mental State Examination (MMSE) ${ }^{1}$ is a widely used brief screening tool for the detection of cognitive impair- ment. In spite of its briefness and practical usefulness, it has a couple of important limitations for the early detection of cognitively impaired states. First, MMSE has poor sensitivity in detecting mild cognitive impairment (MCI), ${ }^{2,3}$ an at-risk state

Received: March 4, 2013 Revised: July 22, 2013 Accepted: August 21, 2013 Available online: January 21, 2014

$\triangle$ Correspondence: Dong Young Lee, MD, PhD

Department of Neuropsychiatry, Seoul National University College of Medicine, Seoul National University Hospital, 101 Daehak-ro, Jongno-gu, Seoul 110-744, Republic of Korea

Tel: +82-2-2072-2205, Fax: +82-2-744-7241, E-mail: selfpsy@snu.ac.kr

(a) This is an Open Access article distributed under the terms of the Creative Commons Attribution Non-Commercial License (http://creativecommons.org/licenses/by-nc/3.0) which permits unrestricted non-commercial use, distribution, and reproduction in any medium, provided the original work is properly cited. 
for the development of dementia, ${ }^{4,5}$ although early detection of the pre-dementia state is increasingly important, with high possibilities for the development of disease modifying treatments for Alzheimer's disease (AD) and related dementias. Second, MMSE consists of test items primarily covering orientation, attention, memory and language, and is less sensitive to frontal executive dysfunction. ${ }^{2,6}$ While memory decline is the earliest and most important cognitive deficit in amnestic MCI (aMCI) and $\mathrm{AD}$, frontal executive dysfunction is frequently more prominent than memory or other cognitive deficits in non-amnestic MCI (naMCI) and non-Alzheimer's disease dementia (NAD), especially frontotemporal dementia (FTD) ${ }^{7}$ and vascular dementia (VD). ${ }^{8}$ Therefore, the assessment of frontal executive dysfunction could make a meaningful contribution to the screening of overall MCI and dementia, or naM$\mathrm{CI}$ and NAD.

Verbal Fluency: Animal category test (VF) provides an assessment of semantic fluency by asking subjects to name as many animals as possible within 1 minute. ${ }^{9}$ Semantic fluency has been shown to be reduced in subjects with MCI compared to those who are cognitively normal $(\mathrm{CN}) .{ }^{10}$ Furthermore, semantic fluency involves not only the ability to search semantic memory using categorical rules, but also to look for the executive function required to track prior responses and block intrusions from other semantic categories. ${ }^{11}$ These imply that VF-supplementation has been proposed to improve the MCI and dementia screening ability of MMSE. Nevertheless, the effect of supplementing VF to MMSE score on the accuracy of $\mathrm{MCI}$ and dementia screening was poorly investigated.

In this study, we aimed to investigate whether the supplementation of VF performance can improve the screening ability of MMSE for MCI, dementia and their major subtypes.

\section{METHODS}

\section{Subjects}

Study subjects were recruited from a pool of individuals registered in a program for the early detection and management of dementia at four centers located in Seoul, Korea (two public health centers, one senior citizens welfare center and one dementia clinic) from January 2000 to May 2011. In this study, $655 \mathrm{CN}, 366 \mathrm{MCI}$ (282 aMCI; 84 naMCI) and 494 dementia (346 AD; $148 \mathrm{NAD}$ ) individuals living in the community were included (all aged 50 years and older).

A diagnosis of dementia was made according to the criteria of the fourth edition of the Diagnostic and Statistical Manual of Mental Disorders. ${ }^{12}$ AD was diagnosed according to the probable or possible $\mathrm{AD}$ criteria of the National Institute of Neurological and Communication Disorders and Stroke/Alzheimer's Disease and Related Disorders Association
(NINCDS-ADRDA). ${ }^{13} \mathrm{VD}$ was diagnosed according to the probable or possible VD criteria of the National Institute of Neurological Disorders and Stroke-Association Internationale pour la Recherche et l'Enseignement en Neurosciences (NINDS-AIREN). ${ }^{14}$ Dementia with Lewy bodies (DLB) or Parkinson's disease dementia (PDD) was diagnosed according to the DLB consensus criteria, ${ }^{15}$ and FTD was diagnosed according to the FTD consensus criteria. ${ }^{16} \mathrm{MCI}$ was diagnosed according to the current consensus criteria. ${ }^{17}$ All MCI individuals had an overall clinical dementia rating scale $(\mathrm{CDR})^{18}$ of 0.5. All NC subjects received a CDR score of 0 . The exclusion criteria for all subjects included any present serious medical, psychiatric and neurologic disorders which could affect the mental function; the presence of severe behavioral or communication problems which would make a clinical examination difficult; an absence of a reliable informant; and the inability of reading Korean [i.e., inability of reading 10 words in the Word List Memory from the Consortium to Establish a Registry for Alzheimer's disease (CERAD) neuropsychological battery ${ }^{19,20}$. All Individuals with minor physical abnormalities (e.g., diabetes with no serious complications, essential hypertension, mild hearing loss or others) were included. The Institutional Review Board of the Seoul National University Hospital, Korea approved the study, and subjects or their legal representatives gave written informed consent.

\section{Clinical and neuropsychological assessments}

All subjects were examined by psychiatrists with advanced training in dementia research according to the CERAD protocol. ${ }^{19,20}$ The CERAD clinical assessment battery included CDR ${ }^{18}$ Blessed Dementia Scale-Activities of Daily Living (BDS-ADL), general medical examination, neurologic examination, laboratory tests, and brain MRI or computed tomography. The standard administration of the CERAD battery was previously described in detail. ${ }^{19,20}$ Reliable informants were necessarily interviewed to acquire the accurate information regarding the cognitive, emotional and functional changes as well as the medical history of the subjects.

MMSE, VF and other neuropsychological tests (15-item Boston Naming Test, Word List Memory, Word List Recall, Word List Recognition, Constructional Praxis, and Constructional Recall) included in the CERAD neuropsychological battery were applied by experienced clinical psychologists or nurses. The VF-supplemented MMSE (MMSE+VF) score was derived from simply summing the scores of the two tests.

A panel consisting of four psychiatrists with expertise in dementia research made the clinical decisions, including diagnosis and CDR, after reviewing all the available raw data. 


\section{Statistical analysis}

Between-group comparisons for continuous data, including demographic and clinical data, were performed by two-tailed $t$ tests. Categorical data were analyzed by the $\chi^{2}$ test. The receiver operating characteristic (ROC) curve analysis was used to compare the screening accuracy for MCI, dementia and their subtypes between MMSE and MMSE+VF. The area under the curve (AUC) of the ROC curve was compared by using the method of Hanley and McNeil. ${ }^{21}$

The level of statistical significance was set as two-tailed

Table 1. Demographic and clinical characteristics of subjects $(\mathrm{N}=1,515)$

\begin{tabular}{|c|c|c|c|c|c|c|c|}
\hline & \multirow{3}{*}{$\begin{array}{c}\mathrm{CN} \\
\mathrm{N}=655\end{array}$} & \multirow{3}{*}{$\begin{array}{c}\mathrm{MCI} \\
\mathrm{N}=366\end{array}$} & \multirow{3}{*}{$\begin{array}{c}\mathrm{D} \\
\mathrm{N}=494\end{array}$} & \multirow{3}{*}{$\begin{array}{c}\mathrm{MCI}+\mathrm{D} \\
\mathrm{N}=860\end{array}$} & \multicolumn{3}{|c|}{$\mathrm{p}$-value } \\
\hline & & & & & $\mathrm{CN}$ vs. $\mathrm{MCI}$ & CN vs. D & $\mathrm{CN}$ vs. $\mathrm{MCI}+\mathrm{D}$ \\
\hline & & & & & $\mathrm{N}=1,021$ & $\mathrm{~N}=1,149$ & $\mathrm{~N}=1,515$ \\
\hline Age (years)* & $69.5 \pm 7.5$ & $71.1 \pm 6.8$ & $72.0 \pm 8.0$ & $71.6 \pm 7.6$ & $<0.001$ & $<0.001$ & $<0.001$ \\
\hline Education (years)* & $7.9 \pm 5.2$ & $8.3 \pm 5.0$ & $8.2 \pm 5.3$ & $8.3 \pm 5.2$ & 0.283 & 0.317 & 0.216 \\
\hline Women $(\%)^{\dagger}$ & 65.0 & 72.1 & 62.3 & 66.5 & 0.020 & 0.353 & 0.584 \\
\hline $\operatorname{CDR}(\%)^{\dagger}$ & & & & & $<0.001$ & $<0.001$ & $<0.001$ \\
\hline CDR 0 (\%) & 100.0 & 0.0 & 0.0 & 0.0 & & & \\
\hline CDR $0.5(\%)$ & 0.0 & 100.0 & 39.7 & 65.1 & & & \\
\hline CDR $1(\%)$ & 0.0 & 0.0 & 47.4 & 27.3 & & & \\
\hline CDR 2+ (\%) & 0.0 & 0.0 & 13.0 & 7.4 & & & \\
\hline MMSE* & $25.3 \pm 3.2$ & $23.0 \pm 4.0$ & $16.3 \pm 5.7$ & $19.2 \pm 6.0$ & $<0.001$ & $<0.001$ & $<0.001$ \\
\hline $\mathrm{VF}^{*}$ & $14.1 \pm 4.0$ & $11.4 \pm 3.8$ & $7.5 \pm 4.0$ & $9.2 \pm 4.3$ & $<0.001$ & $<0.001$ & $<0.001$ \\
\hline
\end{tabular}

Data are presented as mean \pm SD or number (\%). *by Student t-test, $\mathrm{df}=1,019 ; 1,147 ; 1,513$, tby $\chi^{2}$ test, $\mathrm{df}=1 ; 3 ; 3$. CN: cognitively normal, MCI: mild cognitive impairment, D: dementia, MCI+D: overall cognitive impairment including MCI and dementia, CDR: clinical dementia rating, MMSE: mini-mental state examination, VF: verbal fluency using animal category

Table 2. Area under the curves (AUC) and cutoff scores of MMSE, VF, and MMSE+VF in CN, dementia and overall cognitive impairment ( $\mathrm{MCl}+$ dementia) groups

\begin{tabular}{|c|c|c|c|}
\hline & $\mathrm{CN}$ vs. $\mathrm{MCI}(\mathrm{N}=1,021)$ & $\mathrm{CN}$ vs. dementia $(\mathrm{N}=1,149)$ & $\mathrm{CN}$ vs. $\mathrm{MCI}+$ dementia $(\mathrm{N}=1,515)$ \\
\hline \multicolumn{4}{|l|}{ MMSE } \\
\hline AUC & 0.68 & $0.92^{\dagger}$ & 0.82 \\
\hline SE & 0.02 & 0.01 & 0.01 \\
\hline $95 \% \mathrm{CI}$ & $0.65-0.71$ & $0.90-0.93$ & $0.80-0.84$ \\
\hline Cut off & $25 / 26$ & $21 / 22$ & $23 / 24$ \\
\hline Sen/Spe & $73.5 / 55.4$ & $79.8 / 88.9$ & $73.6 / 72.7$ \\
\hline \multicolumn{4}{|l|}{ VF } \\
\hline AUC & 0.69 & 0.88 & 0.80 \\
\hline SE & 0.02 & 0.01 & 0.01 \\
\hline $95 \% \mathrm{CI}$ & $0.66-0.72$ & $0.86-0.90$ & $0.78-0.82$ \\
\hline Cut off & $12 / 13$ & $10 / 11$ & $11 / 12$ \\
\hline Sen/Spe & $64.8 / 60.2$ & $78.3 / 81.7$ & $72.0 / 72.2$ \\
\hline \multicolumn{4}{|l|}{ MMSE+VF } \\
\hline AUC & $0.72^{* \dagger}$ & $0.93^{* \dagger}$ & $0.84^{* \dagger}$ \\
\hline SE & 0.02 & 0.01 & 0.01 \\
\hline $95 \%$ CI & $0.69-0.75$ & $0.92-0.95$ & $0.82-0.86$ \\
\hline Cut off & $37 / 38$ & $33 / 34$ & $35 / 36$ \\
\hline Sen/Spe & $69.4 / 64.6$ & $87.7 / 84.1$ & $77.3 / 75.1$ \\
\hline
\end{tabular}

*significantly greater than that of MMSE, ${ }^{\dagger}$ significantly greater than that of VF (tested by Hanley and McNeil's method). MMSE: Mini-Mental State Examination, VF: verbal fluency using animal category, MMSE+VF: Sum of MMSE and VF, CN: cognitively normal, MCI: mild cognitive impairment, MCI+dementia: overall cognitive impairment including MCI and dementia, AD: Alzheimer's disease, NAD: non-Alzheimer's disease dementia, SE: standard error, CI: confidence interval, Sen/Spe: sensitivity/specificity 


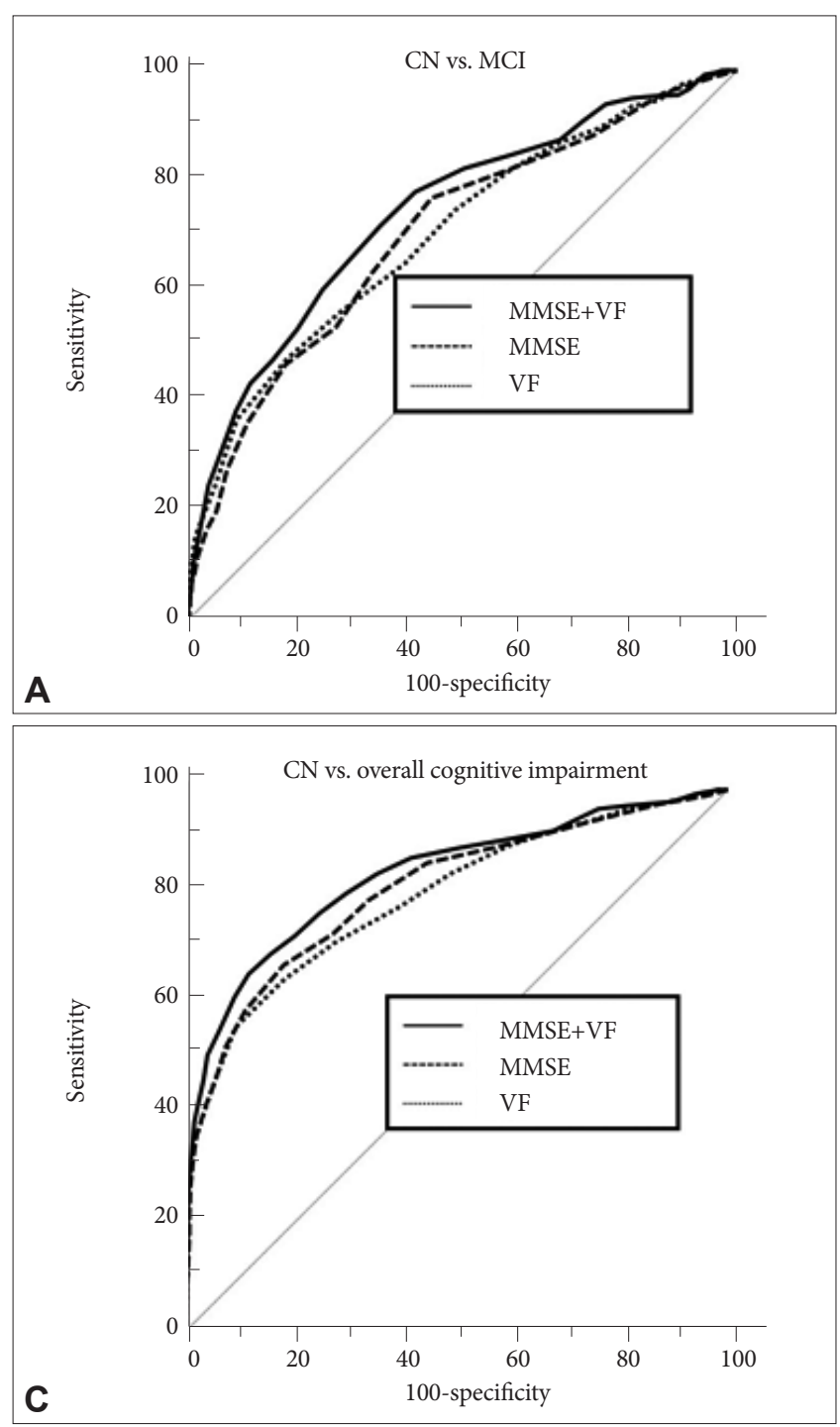

$\mathrm{p}<0.05$. ROC curve analyses were performed by using MedCalc for Windows, version 12.1 (MedCalc Software, Mariakerke, Belgium). All other analyses, except the ROC curve analysis, were performed by using SPSS software, version 15.0 (SPSS Inc, Chicago, IL).

\section{RESULTS}

\section{Demographic and clinical characteristics}

The demographic and clinical characteristics of subjects are summarized in Table 1 .

\section{ROC analysis}

The ROC curve was constructed for each score, as shown in Figures 1 and 2, and the AUC for each ROC curve was calculated. AUC, sensitivity, specificity, cutoff points of MMSE, VF and MMSE+VF scores are shown in Table 2 and 3. The results

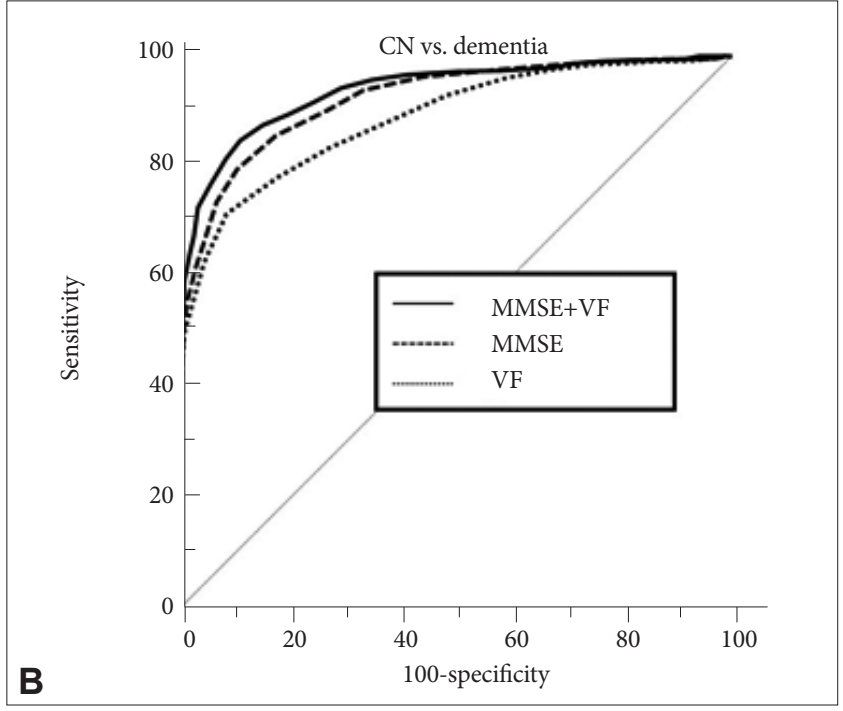

Figure 1. Receiver operating characteristic (ROC) curves of MiniMental State Examination (MMSE), Verbal fluency (VF) and VF-supplemented MMSE (MMSE+VF) in cognitive impairment screening for (A) cognitively normal $(\mathrm{CN})$ versus mild cognitive impairment $(\mathrm{MCl})$, (B) $\mathrm{CN}$ versus dementia and (C) $\mathrm{CN}$ versus overall cognitive impairment (MCl plus dementia).

of the ROC curve comparisons between the MMSE raw score and MMSE+VF score are as follows:

\section{Screening of MCI, dementia and overall cognitive impairment}

MCI screening accuracy of MMSE+VF was significantly better than that of MMSE ( $\mathrm{z}=3.486, \mathrm{p}=0.0005)$ (Table 2, Figure 1A). MMSE+VF showed a significantly superior dementia screening accuracy to MMSE $(\mathrm{z}=2.881, \mathrm{p}=0.0040)$ (Table 2, Figure 1B). MMSE+VF also showed a significantly superior overall cognitive impairment screening accuracy to MMSE $(\mathrm{z}=4.046, \mathrm{p}=0.0001)$ (Table 2, Figure 1C).

\section{Screening the subtypes of MCI and dementia}

MMSE+VF showed a significantly better screening accuracy for both aMCI and naMCI compared to the MMSE alone $(\mathrm{z}=2.992, \mathrm{p}=0.0028$ for $\mathrm{aMCI} ; \mathrm{z}=2.387, \mathrm{p}=0.0170$ for naMCI) 
Table 3. Area under the curves (AUC) and cutoff scores of MMSE, VF and MMSE+VF in CN and subtypes of MCl and dementia

\begin{tabular}{|c|c|c|c|c|}
\hline & $\mathrm{CN}$ vs. $\mathrm{MCI}(\mathrm{N}=937)$ & $\mathrm{CN}$ vs. naMCI $(\mathrm{N}=739)$ & $\mathrm{CN}$ vs. $\mathrm{AD}(\mathrm{N}=1,001)$ & $\mathrm{CN}$ vs. $\mathrm{NAD}(\mathrm{N}=803)$ \\
\hline \multicolumn{5}{|l|}{ MMSE } \\
\hline AUC & 0.70 & 0.59 & $0.93^{\dagger}$ & 0.89 \\
\hline SE & 0.02 & 0.03 & 0.01 & 0.02 \\
\hline $95 \% \mathrm{CI}$ & $0.67-0.73$ & $0.56-0.63$ & $0.91-0.94$ & $0.87-0.91$ \\
\hline Cut off & $25 / 26$ & $25 / 26$ & $21 / 22$ & $21 / 22$ \\
\hline Sen/Spe & $77.0 / 55.4$ & $61.9 / 55.4$ & $82.4 / 88.9$ & $73.7 / 88.9$ \\
\hline \multicolumn{5}{|l|}{ VF } \\
\hline AUC & 0.70 & $0.67^{*}$ & 0.87 & $0.91^{*}$ \\
\hline SE & 0.02 & 0.03 & 0.01 & 0.01 \\
\hline $95 \% \mathrm{CI}$ & $0.67-0.73$ & $0.63-0.70$ & $0.85-0.89$ & $0.89-0.93$ \\
\hline Cut off & $12 / 13$ & $12 / 13$ & $10 / 11$ & $10 / 11$ \\
\hline Sen/Spe & $65.3 / 60.2$ & $63.1 / 60.2$ & $76.6 / 81.7$ & $82.4 / 81.7$ \\
\hline \multicolumn{5}{|l|}{ MMSE+VF } \\
\hline AUC & $0.74^{* \dagger}$ & $0.65^{*}$ & $0.93^{\dagger}$ & $0.93^{* \dagger}$ \\
\hline SE & 0.02 & 0.03 & 0.01 & 0.01 \\
\hline $95 \% \mathrm{CI}$ & $0.71-0.77$ & $0.61-0.68$ & $0.92-0.95$ & $0.91-0.95$ \\
\hline Cut off & $37 / 38$ & $37 / 38$ & $33 / 34$ & $33 / 34$ \\
\hline Sen/Spe & $72.0 / 64.6$ & $60.7 / 64.58$ & $87.3 / 84.1$ & $88.5 / 84.1$ \\
\hline
\end{tabular}

*significantly greater than that of MMSE, ${ }^{\dagger}$ significantly greater than that of VF (tested by Hanley and McNeil's method). MMSE: Mini-Mental State Examination, VF: verbal fluency using animal category, MMSE+VF: Sum of MMSE and VF, CN: cognitively normal, MCI: mild cognitive impairment, AD: Alzheimer's disease, NAD: non-Alzheimer's disease dementia, SE: standard error, CI: confidence interval, Sen/Spe: sensitivity/ specificity

(Table 3, Figure 2A and B). Among the 84 subjects with naMCI, 9 subjects with a single domain naMCI scored worse on only the VF test. In order to minimize the concern, we performed additional analyses on all subjects, excluding the 9 subjects $(n=1,506)$. In this operational condition, we found that MMSE+VF had a significantly better screening ability for MCI $(z=3.024, p=0.0025)$ and overall cognitive impairment $(\mathrm{z}=3.685, \mathrm{p}=0.0002)$ than MMSE. Regarding the dementia subtypes, MMSE+VF was significantly better than MMSE for NAD screening $(\mathrm{z}=4.186, \mathrm{p}<0.0001)$ (Table 3, Figure 2D), but not for $\mathrm{AD}$ screening $(\mathrm{z}=0.810, \mathrm{p}=0.4182)$ (Table 3, Figure 2C).

\section{DISCUSSION}

We found that MMSE+VF had a significantly better screening ability for MCI, dementia and overall cognitive impairment compared to the MMSE alone. For additional subtype analyses for MCI and dementia, we also found that it showed a significantly better ability than MMSE for both MCI subtypes and NAD screening, but not for AD screening.

MMSE also has poor sensitivity in detecting MCI, and is less sensitive to frontal executive dysfunction. Standish, Molloy, Cunje and Lewis ${ }^{22}$ found that a subtest of fluency (type not specified) was best able to discriminate between $\mathrm{CN}$ and $\mathrm{MCI}$ subjects after controlling for age and education. Saxton et al. ${ }^{23}$ suggested that semantic fluency (fruit category) showed a significant baseline decline in 1.5-5 years prior to the onset of AD. Furthermore, sematic fluency involves various executive functions which support or enable the semantic retrieval processes, such as systematic searching and avoiding perseverations and non-category intrusions. ${ }^{24,25}$ These imply that the supplementation of VF has been proposed to improve the MCI and dementia screening accuracy of MMSE.

In the MCI screening, we performed an ROC curve analysis that showed the AUC of 0.718 for MMSE+VF in spite of the VF-supplementation, which improves the screening ability of MMSE. This finding may be explained by two possibilities. First, this modest level of AUC is influenced by poor sensitivity in detecting MCI by MMSE itself rather than by the VFsupplementation effect. Second, the MCI group is comprised of subjects with very heterogeneous cognitive impairments rather than homogeneously prominent executive deficits.

There is several evidence that executive deficits are an important feature of the cognitive decline as well as episodic memory deficits in $\mathrm{AD}$, and that these deficits typically occur early in the disease, and may be the first non-memory deficits to occur. ${ }^{26,27}$ However, in our study, we discovered the VF-sup- 

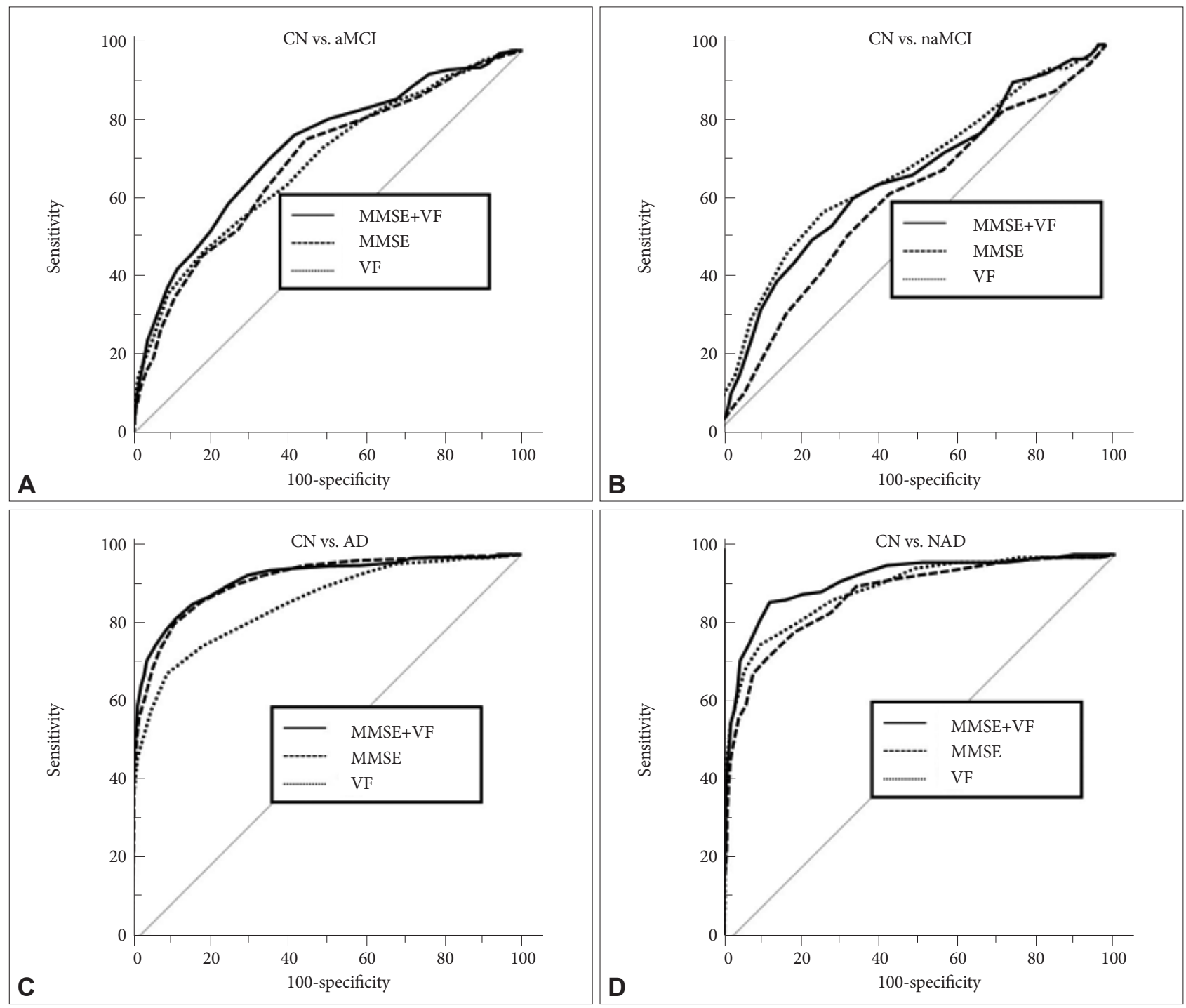

Figure 2. Receiver operating characteristic (ROC) curves of Mini-Mental State Examination (MMSE), Verbal Fluency (VF) and VF-supplemented MMSE (MMSE+VF) in cognitive impairment screening for (A) cognitively normal (CN) versus amnestic mild cognitive impairment (aMCl), (B) CN versus non-amnestic $\mathrm{MCl}$ (naMCl), (C) $\mathrm{CN}$ versus Alzheimer's disease (AD) and (D) CN versus non-Alzheimer's disease dementia (NAD).

plementation effect only for NAD screening, not for $\mathrm{AD}$ screening. This is probably associated with the fact that the frontal executive function is relatively more impaired in NAD, particularly FTD ${ }^{28,29}$ and VD8 compared to AD. Furthermore, the lowered VF-supplementation effect on AD screening in this study is likely to be a reflection of the heterogeneous nature of AD that may include subjects with subtle executive deficits, which may be undetected by VF-supplementation performance.

Several scales have been proposed to overcome the shortcomings of MMSE in MCI and NAD screening by inserting or modified VF, such as the Concise Cognitive Test (CON$\mathrm{COG}){ }^{30}$ the 7-Minutes Screen (7MS) ${ }^{31}$ and the Montreal Cognitive Assessment (MoCA). ${ }^{32}$ Srinivasan30 suggested that
CONCOG has negligible ceiling effects compared with the MMSE. Meulen et al..$^{33}$ demonstrated that the sensitivity of the $7 \mathrm{MS}$ for NAD was $89.4 \%$ with a specificity of $93.5 \%$. Dong et al. ${ }^{34}$ indicated that MoCA is superior to the MMSE in the screening of patients with $\mathrm{MCI}$ who are at a higher risk for incident dementia. Lee et al. ${ }^{35}$ suggested that MoCA had an excellent sensitivity of $89 \%$ and good specificity of $84 \%$ for MCI screening. Though these scales have demonstrated better screening accuracy for MCI or NAD compared with the MMSE, these scales are not widely used in Korean clinical settings due to their unfamiliar westernized items. Therefore, VFsupplementation was selected to improve the screening ability of MMSE for MCI and NAD in our study.

The strengths of this study lead us to believe that our find- 
ings will be replicated in other settings and populations. First, our study population was rather large and had strict diagnoses of CN, MCI, dementia and their subtypes. These were conducted through a clinical evaluation using the strict diagnostic criteria by a panel consisting of four psychiatrists [two panel members (DYL and KWK) were certified as CDR raters by the Memory and Aging Project of the AD Research Center at the Washington University School of Medicine] with expertise in this area. This may have increased the reliability and generalizability of our data. Furthermore, MMSE+VF has an important cost-effect perspective related with the superior MCI and NAD screening accuracy but with a little burden on clinicians. Moreover, it could be broadly applied in some specialized clinical settings (e.g., stroke clinic) with benefits due to its superior NAD screening accuracy.

In conclusion, our findings strongly support the usefulness of VF-supplementation to improve the screening performance of MMSE for MCI and NAD.

\section{Acknowledgments}

This study was supported by a grant from the Korea Healthcare technology R\&D Project, Ministry of Health, Welfare \& Family Affairs, Republic of Korea (Grant No. A092145) and a grant from KIST Open Research Program (Grant No. 2013-1520).

\section{REFERENCES}

1. Folstein MF, Folstein SE, McHugh PR. "Mini-mental state". A practical method for grading the cognitive state of patients for the clinician. J Psychiatr Res 1975;12:189-198.

2. Tombaugh TN, McIntyre NJ. The mini-mental state examination: a comprehensive review. J Am Geriatr Soc 1992;40:922-935.

3. Wind AW, Schellevis FG, Van Staveren G, Scholten RP, Jonker C, Van Eijk JT. Limitations of the Mini-Mental State Examination in diagnosing dementia in general practice. Int J Geriatr Psychiatry 1997;12:101-108.

4. Geslani DM, Tierney MC, Herrmann N, Szalai JP. Mild cognitive impairment: an operational definition and its conversion rate to Alzheimer's disease. Dement Geriatr Cogn Disord 2005;19:383-389.

5. Modrego PJ, Fayed N, Pina MA. Conversion from mild cognitive impairment to probable Alzheimer's disease predicted by brain magnetic resonance spectroscopy. Am J Psychiatry 2005;162:667-675.

6. Royall DR, Mahurin R. EXIT, QED, and DSM-IV: very early Alzheimer's disease. J Neuropsychiatry Clin Neurosci 1994;6:62-65.

7. Gleichgerrcht E, Roca M, Manes F, Torralva T. Comparing the clinical usefulness of the Institute of Cognitive Neurology (INECO) Frontal Screening (IFS) and the Frontal Assessment Battery (FAB) in frontotemporal dementia. J Clin Exp Neuropsychol 2011;33:997-1004.

8. Oguro H, Yamaguchi S, Abe S, Ishida Y, Bokura H, Kobayashi S. Differentiating Alzheimer's disease from subcortical vascular dementia with the FAB test. J Neurol 2006;253:1490-1494.

9. Lezak MD, Howieson DB, Loring DW, Hannay HJ, Fischer JS. Neuropsychological Assessment, 4th Edition. New York: Oxford University Press; 2004.

10. Nutter-Upham KE, Saykin AJ, Rabin LA, Roth RM, Wishart HA, Pare $\mathrm{N}$, et al. Verbal fluency performance in amnestic MCI and older adults with cognitive complaints. Arch Clin Neuropsychol 2008;23:229-241.

11. McDowd J, Hoffman L, Rozek E, Lyons KE, Pahwa R, Burns J, et al. Understanding verbal fluency in healthy aging, Alzheimer's disease, and Parkinson's disease. Neuropsychology 2011;25:210-225.
12. American Psychiatric Association. Diagnostic and Statistical Manual of Mental Disorders. fourth edition. Washington, DC: American Psychiatric Association; 1994.

13. McKhann G, Drachman D, Folstein M, Katzman R, Price D, Stadlan EM. Clinical diagnosis of Alzheimer's disease: report of the NINCDSADRDA Work Group under the auspices of Department of Health and Human Services Task Force on Alzheimer's Disease. Neurology 1984; 34:939-944.

14. Roman GC, Tatemichi TK, Erkinjuntti T, Cummings JL, Masdeu JC, Garcia JH, et al. Vascular dementia: diagnostic criteria for research studies. Report of the NINDS-AIREN International Workshop. Neurology 1993;43:250-260.

15. McKeith IG, Dickson DW, Lowe J, Emre M, O’Brien JT, Feldman H, et al. Diagnosis and management of dementia with Lewy bodies: third report of the DLB Consortium. Neurology 2005;65:1863-1872.

16. Neary D, Snowden JS, Gustafson L, Passant U, Stuss D, Black S, et al. Frontotemporal lobar degeneration: a consensus on clinical diagnostic criteria. Neurology 1998;51:1546-1554.

17. Winblad B, Palmer K, Kivipelto M, Jelic V, Fratiglioni L, Wahlund LO, et al. Mild cognitive impairment--beyond controversies, towards a consensus: report of the International Working Group on Mild Cognitive Impairment. J Intern Med 2004; 256:240-246.

18. Morris JC. The Clinical Dementia Rating (CDR): current version and scoring rules. Neurology 1993;43:2412-2414.

19. Morris JC, Heyman A, Mohs RC, Hughes JP, van Belle G, Fillenbaum $\mathrm{G}$, et al. The Consortium to Establish a Registry for Alzheimer's Disease (CERAD). Part I. Clinical and neuropsychological assessment of Alzheimer's disease. Neurology 1989;39:1159-1165.

20. Lee JH, Lee KU, Lee DY, Kim KW, Jhoo JH, Kim JH, et al. Development of the Korean version of the Consortium to Establish a Registry for Alzheimer's Disease Assessment Packet (CERAD-K): clinical and neuropsychological assessment batteries. J Gerontol B Psychol Sci Soc Sci 2002;57:P47-P53.

21. Hanley JA, McNeil BJ. A method of comparing the areas under receiver operating characteristic curves derived from the same cases. Radiology 1983;148:839-843.

22. Standish TI, Molloy DW, Cunje A, Lewis DL. Do the ABCS 135 short cognitive screen and its subtests discriminate between normal cognition, mild cognitive impairment and dementia? Int J Geriatr Psychiatry 2007;22:189-194.

23. Saxton J, Lopez OL, Ratcliff G, Dulberg C, Fried LP, Carlson MC, et al. Preclinical Alzheimer disease: neuropsychological test performance 1.5 to 8 years prior to onset. Neurology 2004;63: 2341-2347.

24. Kemper S, McDowd J. Dimensions of Cognitive Aging: Executive Function and Verbal Fluency. In: Hofer SM, Alwin DF, Editors. Handbook of Cognitive Aging: Interdisciplinary Perspectives. Thousand Oaks, CA: Sage;2008. p.181.

25. Mayr U, Kliegl R. Complex semantic processing in old age: does it stay or does it go? Psychol Aging 2000;15:29-43.

26. Perry RJ, Watson P, Hodges JR. The nature and staging of attention dysfunction in early (minimal and mild) Alzheimer's disease: relationship to episodic and semantic memory impairment. Neuropsychologia 2000;38:252-271.

27. Amieva H, Phillips LH, Della Sala S, Henry JD. Inhibitory functioning in Alzheimer's disease. Brain 2004;127:949-964.

28. Lipton AM, Ohman KA, Womack KB, Hynan LS, Ninman ET, Lacritz LH. Subscores of the FAB differentiate frontotemporal lobar degeneration from AD. Neurology 2005;65:726-731.

29. Slachevsky A, Villalpando JM, Sarazin M, Hahn-Barma V, Pillon B, Dubois B. Frontal assessment battery and differential diagnosis of frontotemporal dementia and Alzheimer disease. Arch Neurol 2004; 61:1104-1107.

30. Srinivasan S. The concise cognitive test for dementia screening: reliability and effects of demographic variables as compared to the mini mental state examination. Neurol India 2010;58:702-707. 
31. Solomon PR, Hirschoff A, Kelly B, Relin M, Brush M, DeVeaux RD, et al. A 7 minute neurocognitive screening battery highly sensitive to Alzheimer's disease. Arch Neurol 1998;55:349-355.

32. Nasreddine ZS, Phillips NA, Bedirian V, Charbonneau S, Whitehead V, Collin I, et al. The Montreal Cognitive Assessment, MoCA: a brief screening tool for mild cognitive impairment. J Am Geriatr Soc 2005; 53:695-699.

33. Meulen EF, Schmand B, van Campen JP, de Koning SJ, Ponds RW, Scheltens $\mathrm{P}$, et al. The seven minute screen: a neurocognitive screening test highly sensitive to various types of dementia. J Neurol Neurosurg
Psychiatry 2004;75:700-705.

34. Dong Y, Lee WY, Basri NA, Collinson SL, Merchant RA, Venketasubramanian N, et al. The Montreal Cognitive Assessment is superior to the Mini-Mental State Examination in detecting patients at higher risk of dementia. Int Psychogeriatr 2012;24: 1749-1755.

35. Lee JY, Lee DW, Cho SJ, Na DL, Jeon HJ, Kim SK, et al. Brief screening for mild cognitive impairment in elderly outpatient clinic: validation of the Korean version of the Montreal Cognitive Assessment. J Geriatr Psychiatry Neurol 2008; 21:104-110. 